\title{
Dose-Volume Predictors of Radiation Pneumonitis After Lung Stereotactic Body Radiation Therapy (SBRT): Implications for Practice and Trial Design
}

Vitali Moiseenko ${ }^{1}$, Jimm Grimm ${ }^{2}$, Ellen Yorke ${ }^{3}$, Andrew Jackson ${ }^{3}$, Anthony Yip ${ }^{1}$, Minh-Phuong HuynhLe $^{1}$, Anand Mahadevan ${ }^{4}$, Kenneth Forster ${ }^{4}$, Michael T. Milano ${ }^{5}$, Jona A. Hattangadi-Gluth ${ }^{1}$

1. Radiation Medicine and Applied Sciences, University of California San Diego Moores Cancer Center, La Jolla, USA 2. Radiation Oncology, Geisinger Health System, Danville, USA 3. Medical Physics, Memorial Sloan Kettering Cancer Center, New York, USA 4. Radiation Oncology, Geisinger Cancer Institute, Danville, USA 5. Radiology Oncology, Wilmot Cancer Institute, University of Rochester, Rochester, USA

Corresponding author: Vitali Moiseenko, vmoiseenko@health.ucsd.edu

\section{Abstract \\ Background and purpose}

Recently published HyTEC report summarized lung toxicity data and proposed guidelines of mean lung dose (MLD) $<8$ Gy and normal lung receiving at least $20 \mathrm{~Gy}, \mathrm{~V}_{20 \mathrm{~Gy}}<10-15 \%$ to avoid lung toxicity. Support for preferred use of a particular dosimetric parameter has been limited. We performed a detailed dose-volume analysis of data on radiation pneumonitis (RP) following lung stereotactic body radiation therapy (SBRT) to search for parameters showing the strongest correlation with RP.

\section{Materials and methods}

Two patient cohorts (primary and metastatic lung tumor patients) from previously reported studies were analyzed. Total number of patients was 96 , and incidence of grade $\geqslant 2$ RP was $13.5 \%(13 / 96)$. Fitting to the logistic function was performed to investigate correlation between incidence of RP and reported dosimetric and volumetric parameters. Another independent cohort was used to explore correlation between dosimetric parameters.

\section{Results}

Among normal lung parameters (MLD and reported $V_{\mathrm{X}}$ ), only MLD consistently showed significant correlation with incidence of RP. Gross tumor volume (GTV), internal target volume, planning target volume (PTV), and minimum dose covering 95\% of GTV or PTV did not show statistical significance. A significant correlation between reported $\mathrm{V}_{\mathrm{x}}$ and MLD was observed in all cohorts.

Received 08/31/2020

Review began 09/03/2020 Review ended 10/01/2020 Published 10/05/2020

(c) Copyright 2020

Moiseenko et al. This is an open access article distributed under the terms of the Creative Commons Attribution License CC-BY 4.0., which permits unrestricted use, distribution, and reproduction in any medium, provided the original author and source are credited.

\section{Conclusions}

In considering tumor- and target-specific (e.g., GTV, PTV) and normal lung-specific (e.g., MLD, $\mathrm{V}_{\mathrm{X}}$ ) metrics, MLD was the only parameter that consistently correlated with incidence of RP across both cohorts. Because SBRT planning constraints allow small normal lung volumes to receive high doses, utility of MLD is not obvious. The parallel structure of lung is one possible explanation, but correlation between dosimetric parameters obscures elucidation of the preferred or mechanistically based parameter to guide radiotherapy planning.

Categories: Medical Physics, Radiation Oncology

Keywords: stereotactic body radiation therapy, radiation pneumonitis, dose-volume-response

\section{Introduction}

It has been 10 years since the QUANTEC (Quantitative Analyses of Normal Tissue Effects in the Clinic) reports have been published [1]. This effort has been followed by forming two more "TEC" working groups, HyTEC (Hypofractionated/High Dose per fraction), which is near completion with a number of papers published [2-4], and PENTEC (Pediatric Normal Tissue Effects in the Clinic), which is currently in progress [5]. Each working group has been assigned a similar set of tasks: to summarize available literature, to model normal tissue complication probability (NTCP) based upon pooled published data, to provide dosimetric recommendations, and to inform future studies. One of the main challenges has been to find and justify preferred metrics associated with outcomes. This task is particularly challenging not only because the published literature is inconsistent with respect to the scales used to grade toxicities as well as dosimetric parameters analyzed, but also because patient-specific data, which would allow for pooling more granular 
data, are rarely provided [2]. Whenever possible, hypothesis-generating studies to search for preferred metrics are needed to justify radiotherapy planning guidelines.

Stereotactic body radiation therapy (SBRT) with ablative doses has become the standard of care for patients with medically inoperable early-stage non-small cell lung cancer (NSCLC) [6]. Furthermore, the indications for SBRT have been expanded as an option for patients with early-stage, operable NSCLC [7, 8] and lung metastases [9]. Despite advances in SBRT planning and delivery, achieving the desired prescription dose must be balanced against the risk of complications, with lung toxicity being the major limiting factor [2]. Radiation pneumonitis (RP) has been reported in numerous lung SBRT studies [10], with incidence of grade 2 or higher toxicity varying from approximately $10 \%[11,12]$ to as high as $30 \%[13,14]$.

Robust dose-volume predictors of RP are critical in guiding clinical practice and informing lung SBRT clinical trials. Various dosimetric parameters have been tested for correlation with RP, in particular mean lung dose (MLD) [15], or dose-volume cut-offs, Vx (volume of normal lung receiving at least dose ' $\mathrm{x}$ ' Gy) [16]. Commonly, statistical analyses presented in the literature only test if the values of the parameter of choice were different among patients with RP vs without RP, or if a dichotomy can be achieved based on a cut-off value of the parameter of choice. While these observations provide useful data to help guide treatment planning, they do not describe the actual normal tissue dose-volume response. To date, only a few publications have reported correlation, or lack of, between the incidence of pulmonary toxicity and lung SBRT dose-volume parameters, e.g., MLD and V20 [10, 17, 18]. Numerous studies with a goal to explore incidence of RP as a function of various dose metrics have been published for conventional fractionation [19].

Use of Vx cut-offs is appealing because this metric can be readily taken from the dose-volume histogram (DVH). However, as was pointed out in the QUANTEC report on lung toxicity, Vx cut-offs may not be universal and the most predictive values of $\mathrm{x}$ may depend on the treatment technique [19]. Also, because of inter-correlation among Vx parameters [20], statistics do not allow to determine the preferred single predictor which connects with the etiology of toxicity in a meaningful manner. Parameters strongly correlated with such a predictor also become predictors. Hypothesis-generating studies are a pre-requisite to select the preferred metric to guide radiotherapy planning. In this paper, based on the authors' experience with the HyTEC effort, we present detailed dose-volume analyses of data on RP following lung SBRT from two discrete patient cohorts. We explored the incidence of RP as a function of various parameters, specifically normal lung dose-volume metrics and tumor-related metrics. In addition, we tested for correlation between such predictors in the two cohorts and an independently obtained dataset.

\section{Materials And Methods}

\section{Patients}

Two patient cohorts from previously reported studies were used, referenced below as cohorts A [21] and B [22]. These studies were selected because they provided detailed patient-specific data in a tabulated form. In brief, cohort A consisted of 66 (44 male, 22 female) patients with 71 primary (51) or metastatic (20) lung tumors, with planning target volume (PTV) ranging from 9.0 to $100.8 \mathrm{cc}$, who were treated with SBRT with prescribed doses of 40-60 Gy in 5-10 fractions over 5-12 days. Median age was 80 years (58-88 years). Prescription policies were based on the proportion of the prescribed dose covering $80 \%$ of the PTV; the median prescribed dose was $50 \mathrm{~Gy}$ in five fractions. Dose calculation was performed using pencil beam convolution with the Batho method used to account for tissue inhomogeneities. The authors analyzed clinical factors, specifically pulmonary emphysema, tumor location and subclinical interstitial lung disease (ILD) for association with RP (see discussed below). Cohort B consisted of 25 patients ( 21 male, 4 female) with primary (16), recurrent (2) or metastatic (7) lung tumors, PTV ranging from 7.5 to $239.4 \mathrm{~cm}^{3}$, who were treated with SBRT using a prescribed dose of $48 \mathrm{~Gy}$ in 4-8 fractions in 6-7 fields over 5-8 days. Median age was 77 years $(73.8 \pm$ years). Four patients presented with emphysema, three with ILD and one with chronic obstructive pulmonary disease (COPD). Planning goal was that $95 \%$ of PTV receives at least $90 \%$ of the prescribed dose. Collapsed cone convolution method was used for dose calculation. Detailed patient data have been extracted for further analysis. The normal lung parameters tabulated for cohort A were the \% volumes receiving at least $5,10,20$ and $30 \mathrm{~Gy}\left(\mathrm{~V}_{5}, \mathrm{~V}_{10}, \mathrm{~V}_{20}, \mathrm{~V}_{30}\right)$ and the MLD and for cohort $\mathrm{B}$, the \% volumes receiving at least 20,40 and $45 \mathrm{~Gy}\left(\mathrm{~V}_{20}, \mathrm{~V}_{40}, \mathrm{~V}_{45}\right)$, absolute volume in cc receiving at least 24 and 48 $\mathrm{Gy}\left(\mathrm{V}_{\mathrm{cc} 24}\right.$ and $\left.\mathrm{V}_{\mathrm{cc} 48}\right)$, and MLD. Whenever possible, subsets of patients were selected to test for significance of a parameter.

To examine potential correlations between dose-volume parameters in an independent data set, 33 consecutive patients treated with lung SBRT at the University of California San Diego (UCSD) in 2017-2018 were selected. Planning data were extracted from the Eclipse treatment planning system (Varian, Palo Alto, CA). PTV prescription dose was 40-56 Gy in 3-5 fractions. Volumetric-modulated arc therapy (VMAT) with 23 coplanar arcs was used, with a planning goal that at least $95 \%$ of the planning target volume (PTV) receives $100 \%$ of the prescription dose. Analytical anisotropic algorithm, accounting for tissue inhomogeneities, was used to calculate the dose. Gated treatment was used for 12 patients. In planning 4DCT data were sorted into 10 phases, $0-90$ with an increment of 10 , where 0 corresponds to inhale, and 50 to exhale. Phases 30- 
70 or 40-60 centered around exhalation were used to minimize effect of tumor motion. The remaining 21 patients received Gate 100, which means all phases from inhale to exhale are used in planning and dose delivery, and patient is monitored during treatment for breathing irregularities, for example, deep inhale or cough. Normal lung volume was defined as bilateral lung minus internal target volume (ITV). MLD, $\mathrm{V}_{5}, \mathrm{~V}_{13}$ and $\mathrm{V}_{20}$ - parameters commonly used in toxicity studies following SBRT [2] - were extracted. Institutional ethics board approval was obtained to extract, analyze and present the data.

\section{Dose-volume response analysis}

Reported dose-volume parameters for normal lung and tumor-related parameters were analyzed for correlation with incidence of RP grade 2 or higher (grade 2+) and grade 3 or higher (grade $3+$ ) using the Common Terminology Criteria for Adverse Events v5.0 (CTCAE). Specifically, we analyzed MLD and reported $\mathrm{V}_{\mathrm{X}}$ for normal lung dose-volume parameters as well as gross tumor volume (GTV), ITV, and PTV for tumorrelated parameters. For each parameter, fitting to the logistic function was performed; for example, if $\mathrm{V}_{10}$ was explored, the function was:

$$
P=1 /\left[1+\exp \left(-4 \gamma_{50}\left(V_{10} / V_{10,50}-1\right)\right)\right]
$$

where $\mathrm{V}_{50}$ is the normalized slope and $\mathrm{V}_{10,50}$ is the value of $\mathrm{V} 10$ at which $50 \%$ of patients develop RP. Maximum likelihood method was used to obtain model parameter values, $\mathrm{V}_{50}$ and $\mathrm{V}_{10,50}$. Likelihood-profile method was used to calculate 95\% confidence intervals (CI) [23]. A likelihood ratio test was used to establish if the observed dependence significantly $(\mathrm{p}<0.05)$ improves on the null hypothesis assuming no dependence. To calculate likelihood value for the null hypothesis incidence was set to the value averaged over the cohort. Spearman test was used to check for correlation between dose-volume parameters. All tests were two-tailed and $\mathrm{p}<0.05$ was considered statistically significant.

\section{Results}

Total number of patients in the study was 96,13 of whom developed RP (13.5\%). This sample size and toxicity rate was similar to those published in the studies that were summarized in the HyTEC report (see supplemental table in the HyTEC report [2]). Wilcoxon rank sum test showed that MLD was significantly different, $p=0.002$, in patients with vs without grade $2+$ toxicity, thereby justify further analysis for doseresponse. Table 1 shows the results of fitting the dose-volume data to the logistic function for both grade $2+$ and $3+$ toxicity in cohorts A and B. None of the tumor or target volume characteristics (GTV, ITV, PTV) showed statistical significance. Among normal lung parameters (MLD and reported $\mathrm{V}_{\mathrm{X}}$ ), only MLD consistently showed significant correlation with incidence of RP for both cohorts (Figure 1). Specifically, MLD was statistically significant for both grade $2+$ and grade $3+$ in cohort $\mathrm{A}$, as well as grade $2+$ in cohort $\mathrm{B}$. However, this significance was only marginal for grade $3+$ in cohort $B, p=0.058$. 


\section{Cureus}

\begin{tabular}{|c|c|c|c|c|c|c|}
\hline \multirow[b]{2}{*}{ Parameter } & \multicolumn{3}{|l|}{ Grade 2+ } & \multicolumn{3}{|l|}{ Grade 3+ } \\
\hline & $\begin{array}{l}D_{50}, V_{50} \text { or } V_{x, 50}(95 \% \\
C l)\end{array}$ & $V_{50}(95 \% \mathrm{Cl})$ & $\begin{array}{l}\mathrm{p} \text {, likelihood } \\
\text { ratio }\end{array}$ & $\begin{array}{l}D_{50}, V_{50} \text { or } V_{x, 50}(95 \% \\
C l)\end{array}$ & $\gamma_{50}(95 \%$ Cl) & $\begin{array}{l}\mathrm{p} \text {, likelihood } \\
\text { ratio }\end{array}$ \\
\hline & Cohort A [21] & & & & & \\
\hline GTV, cc & 78.2 (at limit) & $0.68(0.41,1.02)$ & 0.390 & $53.6(30.5, \infty)$ & $1.01(0.63,1.57)$ & 0.098 \\
\hline PTV, cc & $162.1(72.3, \infty)$ & $0.74(0.46,1.19)$ & 0.431 & $106.8(68.9, \infty)$ & $1.18(0.66,1.95)$ & 0.097 \\
\hline $\begin{array}{l}\text { GTV D } 95, \\
\text { Gy }\end{array}$ & 80.8 (at limit) & $1.58(-.25,3.87)$ & 0.310 & 91.8 (at limit) & $\begin{array}{l}1.73(-0.71, \\
5.07)\end{array}$ & 0.479 \\
\hline MLD, Gy & $6.4(4.8,88.1)$ & $1.20(0.58,2.05)$ & 0.042 & $6.3(5.0,51.8)$ & $1.74(0.78,3.31)$ & 0.037 \\
\hline$V_{5}, \%$ & $33.3(25.2, \infty)$ & $1.22(0.59,2.08)$ & 0.038 & $33.8(26.4, \infty)$ & $1.69(0.75,3.22)$ & 0.045 \\
\hline$v_{10}, \%$ & $20.4(15.7,69.1)$ & $1.23(0.65,2.03)$ & 0.018 & $20.7(16.4,60.3)$ & $1.76(0.87,3.27)$ & 0.016 \\
\hline$v_{20}, \%$ & 13.4 (at limit) & $0.83(0.37,1.41)$ & 0.294 & 11.1 (at limit) & $1.24(0.57,2.29)$ & 0.159 \\
\hline \multirow[t]{2}{*}{$v_{30}, \%$} & 7.8 (at limit) & $0.84(0.40,1.42)$ & 0.251 & 6.7 (at limit) & $1.24(0.58,2.28)$ & 0.153 \\
\hline & Conort B [22] & & & & & \\
\hline ITV, cc & 1217.0 (at limit) & $\begin{array}{l}0.24(-0.04 \\
0.54)\end{array}$ & 0.975 & at limit & - & - \\
\hline PTV, cc & 368.9 (at limit) & $\begin{array}{l}0.28(-0.06 \\
0.64)\end{array}$ & 0.752 & 681.7 (at limit) & $\begin{array}{l}0.38(-0.01, \\
0.79)\end{array}$ & 0.841 \\
\hline $\begin{array}{l}\text { PTV D } \\
\text { Gy }\end{array}$ & 49.0 (at limit) & $\begin{array}{l}3.38(-3.07 \\
10.24)\end{array}$ & 0.332 & 48.6 (at limit) & $\begin{array}{l}5.92(-1.15 \\
14.00)\end{array}$ & 0.121 \\
\hline MLD, Gy & $5.9(4.5,30.5)$ & $0.99(0.24,2.01)$ & 0.037 & $6.8(5.16, \infty)$ & $1.09(0.28,2.22)$ & 0.058 \\
\hline $\mathrm{V}_{20}, \%$ & 12.1 (at limit) & $\begin{array}{l}0.56(-0.01, \\
1.29)\end{array}$ & 0.244 & 13.8 (at limit) & $0.72(0.08,1.60)$ & 0.237 \\
\hline$v_{40}, \%$ & 5.6 (at limit) & $0.39(0.02,0.84)$ & 0.332 & 6.1 (at limit) & $0.57(0.14,1.11)$ & 0.240 \\
\hline$v_{45}, \%$ & 3.8 (at limit) & $0.36(0.05,0.72)$ & 0.303 & 4.7 (at limit) & $0.49(0.15,0.91)$ & 0.277 \\
\hline $\mathrm{V}_{\mathrm{cc} 24}, \mathrm{cc}$ & $258.6(172.3, \infty)$ & $0.67(0.15,1.38)$ & 0.076 & $301.1(207.1, \infty)$ & $0.82(0.23,1.71)$ & 0.097 \\
\hline \multirow[t]{2}{*}{$\mathrm{V}_{\mathrm{cc} 48}, \mathrm{cc}$} & 112.8 (at limit) & $0.28(0.02,0.58)$ & 0.597 & 984.7 (at limit) & $0.35(0.07,0.68)$ & 0.939 \\
\hline & Combined data & & & & & \\
\hline MLD, Gy & $6.1(5.0-9.0)$ & $1.18(0.73-1.77)$ & $<0.001$ & $6.6(5.5-10.2)$ & $1.47(0.90-2.27)$ & $<0.001$ \\
\hline
\end{tabular}

TABLE 1: Grade 2+ and 3+ lung toxicity, results of fitting to the logistic model. If the likelihoodprofile was flat and did not fall below the cut-off value, maximum log-likelihood minus 1.92, at either lower or upper end, confidence intervals are marked as "at limit". 


\section{Cureus}

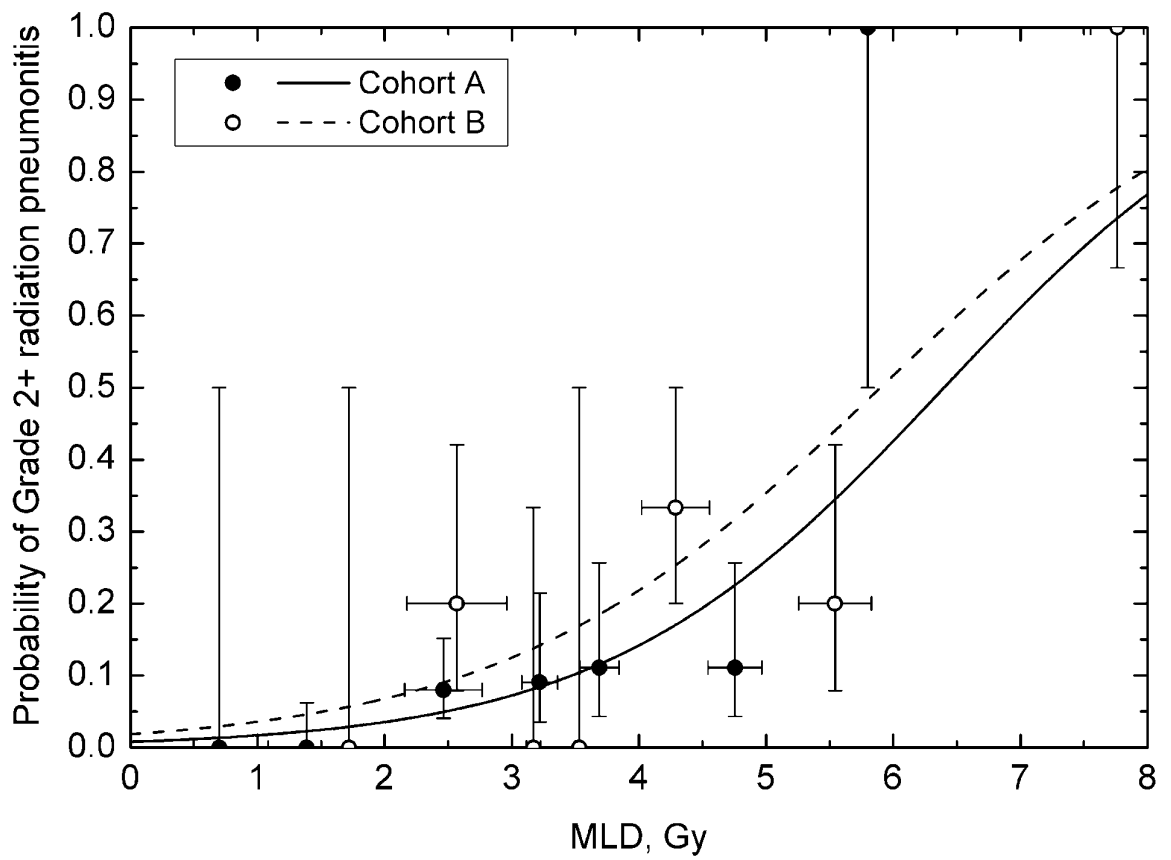

FIGURE 1: Probability of grade 2 or higher radiation pneumonitis as a function of mean lung dose (MLD) for the studied patient cohorts $A$ and B. Vertical error bars are binomial $68 \%$ confidence intervals, horizontal error bars are standard deviations. Bin size was set to $1 \mathrm{~Gy}$, except between 3 and 4 Gy where a 0.5 Gy size was used.

Based on the available dose-volume data, values of $\mathrm{V}_{\mathrm{X}}$ were tested for correlation with incidence of toxicity. Only $\mathrm{V}_{5}$ and $\mathrm{V}_{10}$ in cohort A resulted in a statistically significant $\mathrm{V}_{\mathrm{x}}$-response dependence (Table 1). This held for both grade $2+$ and grade $3+$. Values of $\mathrm{V}_{50}$ and $\mathrm{V}_{\mathrm{x}, 50}$ for $\mathrm{V}_{5}$ and $\mathrm{V}_{10}$, cohort $A$, were very similar for grade $2+$ and grade $3+$ (Table 1). Specifically, $\mathrm{V}_{5,50}$ was $33.3 \%$ for grade $2+$ and $33.8 \%$ for grade $3+$.

$\mathrm{V}_{10,50}$ values were $20.4 \%$ for grade $2+$ and $20.7 \%$ for grade $3+$. Figure 2 shows incidence of RP as a function of $\mathrm{V}_{5}$ and $\mathrm{V}_{10}$ in cohort $\mathrm{A}$. No logistic fit for $\mathrm{V}_{\mathrm{X}}$ was significant in cohort $\mathrm{B}$. 


\section{Cureus}

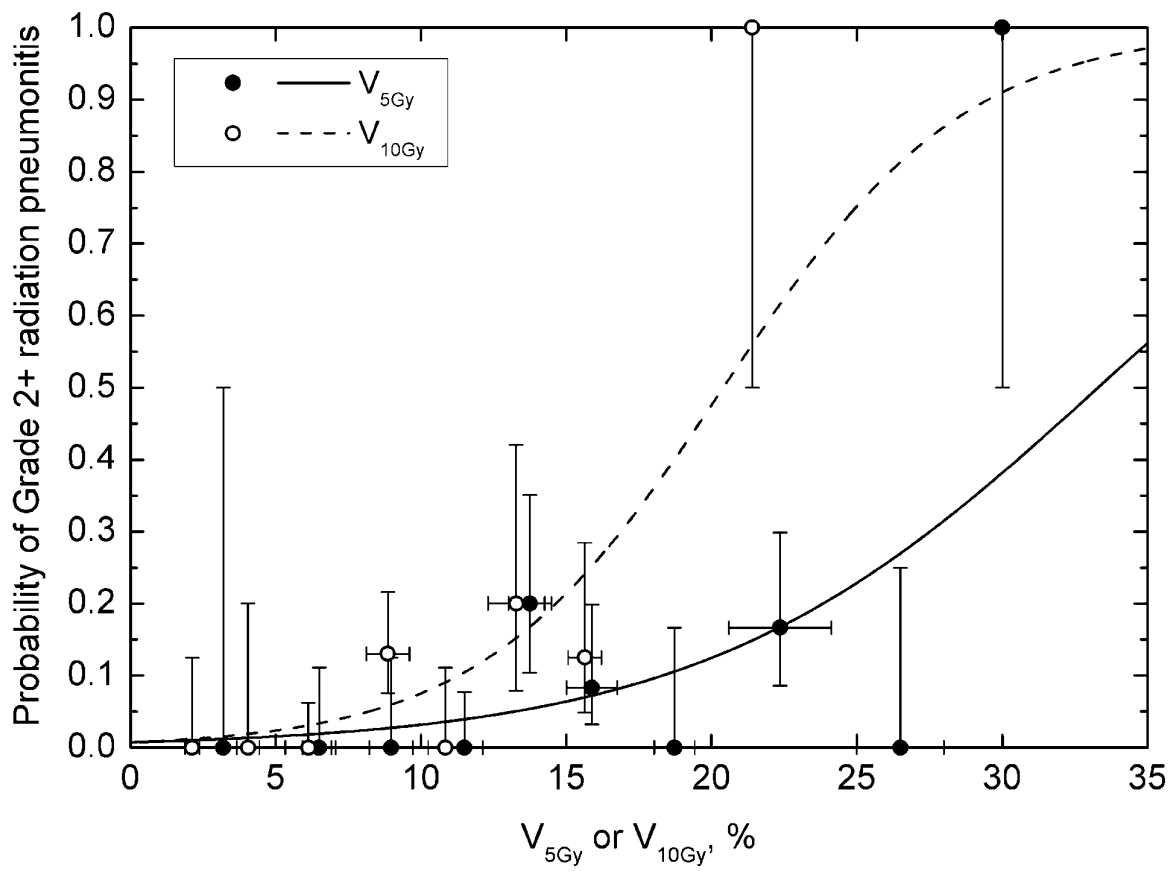

FIGURE 2: Probability of grade 2 or higher radiation pneumonitis as a function of V5 and V10, cohort A. Vertical error bars are binomial $68 \%$ confidence intervals, horizontal error bars are standard deviations. Bin size was set to $2.5 \%$.

MLD data from cohorts A and B were combined and statistically significant dose-response was obtained for both grade $2+$ and grade $3+$ toxicity. Notably, when fitting was performed separately for cohorts A and B, the values of $\mathrm{D}_{50}$ were very close, albeit with broad CI (Table 1 ). Figure 3 shows dose-response curves and combined data. Model parameters are shown in the bottom row of Table 1.

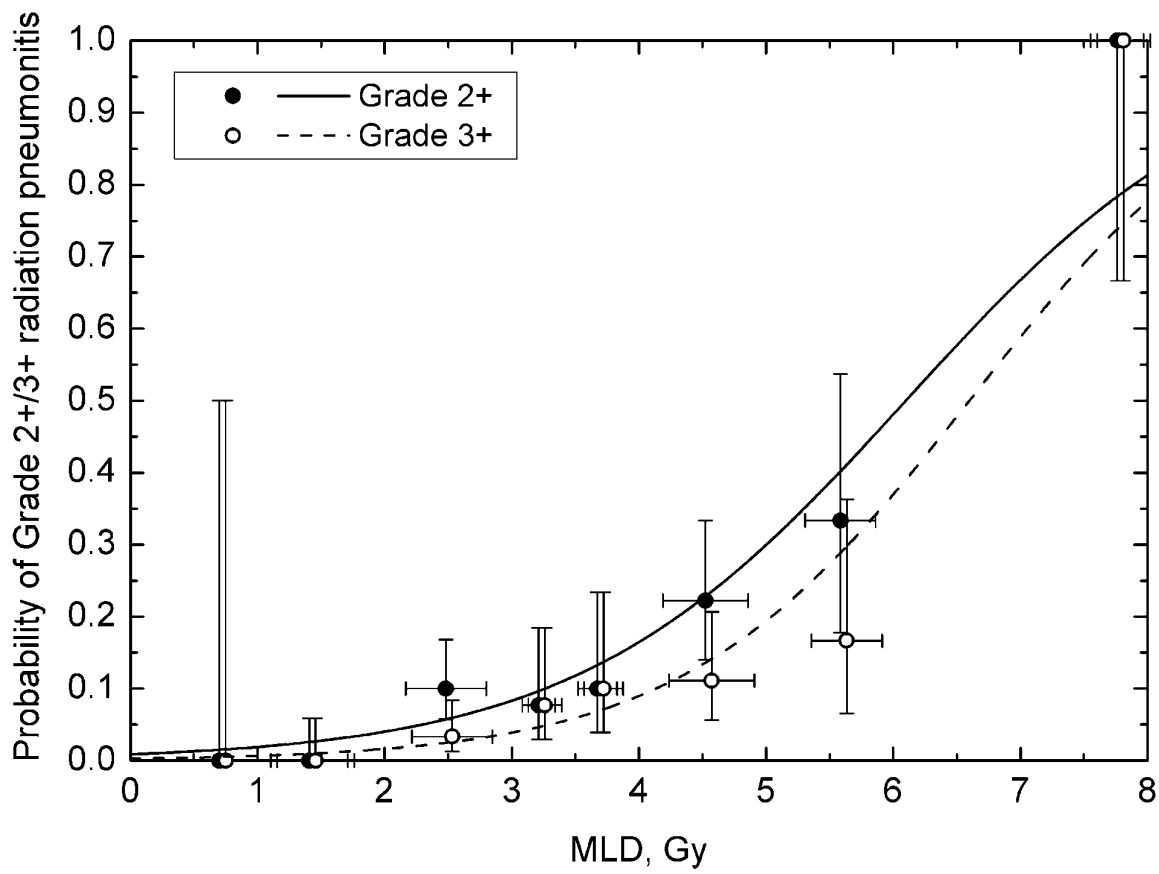

FIGURE 3: Probability of grade 2 or higher, and grade 3 or higher as a 


\section{Cureus}

function of mean lung dose (MLD) for the combined data. Vertical error bars are binomial $68 \%$ confidence intervals, horizontal error bars are standard deviations. Bin size was set to $1 \mathrm{~Gy}$, except between 3 and 4 Gy where a 0.5 Gy size was used.

Table 2 shows the correlations between the quantity in the "Parameter" column with normal lung MLD and $\mathrm{V}_{20}$; the latter has been reported most broadly in the literature [10]. Spearman's rank-order correlation coefficients showed that both $\mathrm{V}_{5}$ and $\mathrm{V}_{10}$ strongly correlate with MLD in cohort A, Rs $=0.936$ and 0.966 , respectively. Overall, there was statistically significant correlation between all $\mathrm{Vx}$ as a percent volume and MLD, as well as between $V_{x}$ and $V_{20}$. Correlation was weaker for tumor-specific parameters and insignificant for PTV $D_{95}$, cohort B. The correlations were also tested in the UCSD independent validation cohort. Figure 4 shows strong correlation between $\mathrm{V}_{5}, \mathrm{~V}_{13}, \mathrm{~V}_{20}$ and MLD for the UCSD cohort with large and significant Spearman's rank-order correlation coefficients, (Rs $\geqslant 0.931, \mathrm{p}<0.001$ ). Effect of possible confounders, specifically pulmonary emphysema, tumor location (upper/middle vs lower) and ILD was studied in the original report for the cohort A [21]. ILD was the only factor significantly associated with RP. In the cohort B [22] one patient had COPD, four had emphysema, tumor location was peripheral in 21 of 25 patients and peripheral/central in remaining four. This is insufficient to perform statistical analyses to assess effect of possible confounders.

\begin{tabular}{|c|c|c|c|c|c|}
\hline \multicolumn{3}{|l|}{ Cohort A [21] } & \multicolumn{3}{|l|}{ Cohort B [22] } \\
\hline Parameter & MLD, Gy & Lung $\mathrm{V}_{20}, \%$ & Parameter & MLD Gy & $v_{20}$ \\
\hline GTV, cc & $0.643^{* \star *}$ & $0.667^{\star \star \star}$ & ITV, cc & $0.583^{\star \star}$ & $0.654^{\star \star \star *}$ \\
\hline PTV, cc & $0.623^{* \star \star x}$ & $0.650^{\star \star *}$ & PTV, cc & $0.556^{\text {*t }}$ & $0.627^{\times \times \star}$ \\
\hline GTVD D95, Gy & $0.376^{\star *}$ & $0.398^{\star \star *}$ & $\mathrm{PTV}_{95}$ Gy & 0.053 & 0.169 \\
\hline$V_{5}, \%$ & $0.936^{\star * *}$ & $0.783^{* \star *}$ & $v_{20}, \%$ & $0.764^{* \star \star}$ & - \\
\hline$v_{10}, \%$ & $0.966^{* \star *}$ & $0.914^{* \star \star}$ & $V_{40}, \%$ & $0.621^{\star \star \star}$ & $0.679^{\star \star \star x}$ \\
\hline$v_{20}, \%$ & $0.927^{\star \star \star}$ & - & $v_{45}, \%$ & $0.593^{\text {*t }}$ & $0.612^{\star \star x}$ \\
\hline$V_{30} \%$ & $0.914^{\star \star \star}$ & $0.984^{* \star \star}$ & MLD Gy & - & $0.764^{\star \star x+}$ \\
\hline \multirow[t]{2}{*}{ MLD, Gy } & - & $0.927^{\star \star *}$ & $V_{24}, c c$ & $0.514^{\star \star}$ & $0.602^{\star \star \star}$ \\
\hline & & & $V_{48}, \mathrm{cc}$ & $0.487^{\star}$ & $0.476^{*}$ \\
\hline
\end{tabular}

TABLE 2: Spearman's rank-order correlation coefficients for the considered tumor and lungspecific parameters.

${ }^{*} 0.01 \leq p<0.05 ;{ }^{* *} 0.001 \leq p<0.01 ;{ }^{* * \star} p<0.001$ 


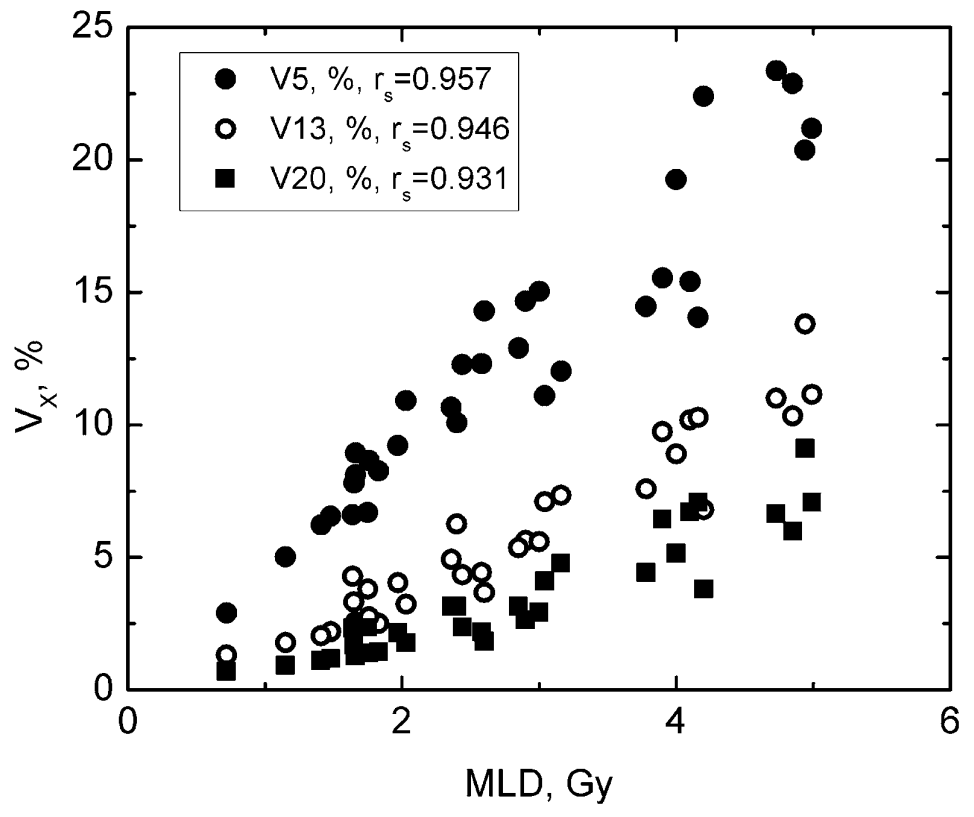

FIGURE 4: Scatterplot showing correlations between mean lung dose (MLD) and V5, V13 and V20. Insert shows Spearman's rank-order correlation coefficients.

Incidence of radiation-induced lung toxicity as a function of MLD was separately assessed for patients with and without underlying interstitial lung disease (ILD). In Cohort A, 11 patients had subclinical ILD before SBRT as did at least three in Cohort B. However, because of the small number of patients with toxicities, best values were only obtained for patients with ILD in the Cohort A, grade 2+ toxicity. While $95 \%$ CI were not established, it was possible to calculate $68 \% \mathrm{CI}$. Obtained logistic model parameters were indeed indicative of substantially higher sensitivity, $D_{50}=3.7 \mathrm{~Gy}(68 \% \mathrm{CI} 2.7-5.1 \mathrm{~Gy})$, and $\mathrm{Y}_{50}=0.73(0.22-1.34)$ as opposed to $\mathrm{D}_{50}$ of $6.1 \mathrm{~Gy}, \mathrm{\gamma}_{50}$ of 1.18 for combined cohort, Grade 2+ model. This observation, however, must be interpreted with caution as the dose-response was not statistically significant.

\section{Discussion}

Developing evidence-based dose-volume metrics to generate guidelines for normal tissue sparing is a challenging task assigned to working groups, such as QUANTEC [1], HyTEC [2, 3] or PENTEC [5]. Selected metrics have to be justified on statistical and mechanistic merits and hypothesis-generating studies taking advantage of detailed patient-specific data provide this justification. Currently, both Vx and MLD are commonly used dosimetric correlates for lung toxicity following SBRT $[2,19]$. Supplementary Table 1 in the HyTEC report on lung toxicity following SBRT [2] shows 16 publications where lung toxicity correlated with either $\mathrm{V}_{5}, \mathrm{~V}_{10}, \mathrm{~V}_{13}, \mathrm{~V}_{20}$ or MLD, with $\mathrm{V}_{20}$ and MLD being the most popular choices attempted in 15 publications.

In considering tumor- and target-specific (e.g., GTV, PTV) and normal lung-specific (e.g., MLD, $\mathrm{V}_{\mathrm{x}}$ ) metrics, our results showed that MLD was the only parameter that consistently correlated with incidence of RP across both cohorts A and B, as well as in the combined cohort. Proponents for MLD argue that regional lung function is compromised at any dose, with the underlying assumption that local injury is linearly dependent on dose [24]. Local dose-response for perfusion loss has been demonstrated in SBRT patients [25]. Studies of changes in regional lung perfusion as a function of conventional fractionated doses have shown that this dependence may follow a sigmoid relationship, with no loss of perfusion below 10-15 Gy and a plateau at about 55 Gy [24]. Although this deviates from the linear dependence theory, the authors deemed MLD as a reasonable surrogate for overall response parameter, which is a sum of regional injuries. This implies mechanistically-based use of MLD in SBRT and emphasizes the need to understand the local lung function (ventilation and perfusion) before SBRT. In theory, if there is a threshold dose beyond which function is $100 \%$ compromised, it will be lower for SBRT because using Radiation Biologically Effective Dose (BED) Calculator, $60 \mathrm{~Gy} / 30$ has BED(3) of $100 \mathrm{~Gy}$, which is the same BED(3) of 31.9Gy/5, assuming $\alpha / \beta=3 \mathrm{~Gy}$. 
threshold dose and that toxicity is avoided if a minimum total lung volume is undamaged. Unfortunately, data for conventional fractionation argues against the existence of a single, or even narrowly distributed threshold. Indeed, the QUANTEC report showed a broad range in threshold doses $\left(\mathrm{V}_{5}\right.$ to $\left.\mathrm{V}_{40}\right)$ correlating to incidence of RP while also noting two important findings: 1) different $\mathrm{V}_{\mathrm{X}}$ parameters correlate with one another and MLD; 2) use of $\mathrm{V}_{\mathrm{X}}$ cut-offs has to be noticed as they may not be transferrable between radiotherapy techniques [19]. Supporting this first finding, we also saw significant correlation between $\mathrm{V}_{5}$, $\mathrm{V}_{13}, \mathrm{~V}_{20}$ and MLD, $\mathrm{p}<0.001$, in our independent cohort. While this makes it impossible based on statistical analysis alone to single out the best predictor, it also means that, at least in a single institution setting, using MLD is as good as any other metric. Advanced statistical methods, for example Lasso or elastic nets regularized regression allow us to address the problem of co-linearity. This complex problem which requires large sets of detailed data has been gaining attention in the literature.

Robustness of MLD may be questioned because dose alone is insufficient to describe biological effect and accounting for dose-per-fraction may be needed, in particular for normal tissues. Notably, in this study physical dose without corrections for dose-per-fraction such as biologically equivalent dose in 2 Gy fractions (EQD2), was used for both the dose in $\mathrm{V}_{\mathrm{x}}$ and MLD. MLD was the strongest predictor despite a broad range in the number of fractions in the two cohorts. This provides the simplest way to guide treatment planning but also raises a question as to whether this is also a consequence of statistical correlation. Specifically, if MLD defined as physical dose and mean dose calculated following conversion into EQD2 are correlated, finding preferred metric will be challenging. In this study we were restricted to what was reported. Conversion of MLD to mean EQD2 is non-linear and cannot be performed without full DVH data. This is a limitation in our study and conversion to common fractionation, for example EQD2 is encouraged.

Variations of the volume cut-off approach, specifically absolute volume spared, have been put forward [26] and these may provide connection with a mechanistic nature of development of RP. For example, lung dosethresholds used in the RTOG 0915 trial required that less than 1500 and $1000 \mathrm{cc}$ of total lung (minus PTV) receive a specified dose, to minimize risks of pulmonary function decline and pneumonitis, respectively. Similar thresholds were included in the American Association of Physicists in Medicine Task Group 101 report, which they describe as "minimum critical volume below threshold" [26]. Spared volume style constraints have been used for other parallel tissues, such as liver [3], though further investigation of their validity is needed.

Considering biological mechanisms, published studies propose various pro-inflammatory cytokines that contribute to the acute inflammation and late fibrosis seen in radiation pneumonitis. This inflammatory cascade has several well-recognized triggers including DNA double-strand breaks, endothelial cell damage, free radical damage, upregulation of leukocyte adhesion molecules and platelet derived growth factor (PDGF) isoforms, activation of type II pneumocytes, and hypoxia [27, 28]. The existence of several cascade triggers supports the theory that inflammation follows a linearly dose dependent response rather than the incapacitation hypothesis underlying the $\mathrm{V}_{\mathrm{X}}$ approach. On this basis, use of MLD is supported as it fundamentally parallels the biological inflammatory dose-response.

Decline in pulmonary function after SBRT is a potential risk, though the extent to which this occurs is unclear. As some studies suggest no or minimal decline in pulmonary function, and tumor progression can be life threatening, baseline pulmonary functional status may not be a major factor in decision making with respect to SBRT dose-fractionation. With some studies suggesting a progressive decline in diffusion capacity, risk of pulmonary function decline should be discussed with the patient, though such changes can occur as part of the natural course of chronic obstructive pulmonary disease (COPD). In patients without COPD, diffusion capacity could potentially improve after SBRT. In contrast, patients with interstitial lung disease, regardless of measured pulmonary function, are at high risk of life-threatening toxicity after SBRT [2], and these risks must be weighed against potential benefits. Deferring treatment, or use of more stringent lung dosimetry should be considered.

Patient- and disease-specific parameters, for example age, sex, tumor location, performance status, may also play a role in development of RP [2]. Effect of age, sex, tumor location and performance status were investigated for correlation with RP in one of the source publications [21]. None of these factors were statistically significant with respect to predicting RP risks. Notably, another study [29] demonstrated that the incidence of RP can be minimized if patients are screened for radiographic evidence of ILD [15, 22] and high values of biological markers (KL-6 and SP-D) before treatment. Increased treatment-related toxicity in patients with co-existing interstitial lung disease who receive SBRT has been noted in a comprehensive review [30]. Small sample size as well as relatively low toxicity rates were further limitations of our study. This, unfortunately, is a common problem with outcomes data reporting. Detailed patient-specific data are typically not available for large cohorts.

\section{Conclusions}

Our findings show that there is a strong statistical basis for using MLD as a predictor of RP within single institutions. These findings are also supported by mechanistic considerations. The relatively simple MLD 
metric has implications for clinical practice and lung SBRT clinical trials, especially in combination with immunotherapy (trials NCT03867175 and NCT03436056). While validation is required with data from different institutions, MLD is promising for use across institutions and in cooperative group trials. Limiting bilateral MLD $<8$ Gy was recommended in the HyTEC report for fractionated SBRT to ensure safety. Our study provides further justification for using MLD to predict RP risks. Competing Vx and critical volume (i.e., spared lung) approaches may have promise, but need further validation. Our main conclusion is simply that MLD may have merit for SBRT, and it should at least be considered along with the more common $\mathrm{V}_{\mathrm{x}}$ and critical volume metrics in future studies.

\section{Additional Information \\ Disclosures}

Human subjects: All authors have confirmed that this study did not involve human participants or tissue. Animal subjects: All authors have confirmed that this study did not involve animal subjects or tissue. Conflicts of interest: In compliance with the ICMJE uniform disclosure form, all authors declare the following: Payment/services info: All authors have declared that no financial support was received from any organization for the submitted work. Financial relationships: All authors have declared that they have no financial relationships at present or within the previous three years with any organizations that might have an interest in the submitted work. Other relationships: All authors have declared that there are no other relationships or activities that could appear to have influenced the submitted work.

\section{Acknowledgements}

This work was partially supported by the National Institutes of Health (NIH) grants \# TL1TR001443 (AY) and R01CA238783-01 (JAH-G). The content is solely the responsibility of the authors and does not necessarily represent the official views of the NIH.

\section{References}

1. Marks LB, Ten Haken RK, Martel MK: Guest editor's introduction to QUANTEC: a users guide . Int J Radiat Oncol Biol Phys. 2010, 76:1-2. 10.1016/j.ijrobp.2009.08.075

2. Kong FS, Moiseenko V, Zhao J, et al.: Organs at risk considerations for thoracic stereotactic body radiation therapy: what is safe for lung parenchyma? (IN PRESS). Int J Radiat Oncol Biol Phy. 2018, 10.1016/j.ijrobp.2018.11.028

3. Miften M, Vinogradskiy Y, Moiseenko V, et al.: Radiation dose-volume effects for liver SBRT (IN PRESS). Int J Radiat Oncol Biol Phys. 2018, 10.1016/j.ijrobp.2017.12.290

4. Milano MT, Grimm J, Soltys SG, et al.: Single- and multi-fraction stereotactic radiosurgery dose tolerances of the optic pathways (IN PRESS). Int J Radiat Oncol Biol Phys. 2018, 10.1016/j.ijrobp.2018.01.053

5. Constine LS, Ronckers CM, Hua CH, Olch A, Kremer LCM, Jackson A, Bentzen SM: Pediatric normal tissue effects in the clinic (PENTEC): an international collaboration to analyse normal tissue radiation dosevolume response relationships for paediatric cancer patients. Clin Oncol. 2019, 31:199-207. 10.1016/j.clon.2019.01.002

6. de Jong EEC, Guckenberger M, Andratschke N, et al.: Variation in current prescription practice of stereotactic body radiotherapy for peripherally located early stage non-small cell lung cancer: recommendations for prescribing and recording according to the ACROP guideline and ICRU report 91 . Radiother Oncol. 2020, 142:217-223. 10.1016/j.radonc.2019.11.001

7. Timmerman R, Galvin J, Michalski J, et al.: Accreditation and quality assurance for Radiation Therapy Oncology Group: multicenter clinical trials using stereotactic body radiation therapy in lung cancer. Acta Oncol. 2006, 45:779-786. 10.1080/02841860600902213

8. Davis JN, Medbery C 3rd, Sharma S, et al.: Stereotactic body radiotherapy for early-stage non-small cell lung cancer: clinical outcomes from a National Patient Registry. J Radiat Oncol. 2015, 4:55-63. 10.1007/s13566014-0177-0

9. Rusthoven KE, Hammerman SF, Kavanagh BD, Birtwhistle MJ, Stares M, Camidge DR: Is there a role for consolidative stereotactic body radiation therapy following first-line systemic therapy for metastatic lung cancer? A patterns-of-failure analysis. Acta Oncol. 2009, 48:578-583. 10.1080/02841860802662722

10. Zhao J, Yorke ED, Li L, et al.: Simple factors associated with radiation-induced lung toxicity after stereotactic body radiation therapy of the thorax: a pooled analysis of 88 studies. Int J Radiat Oncol Biol Phys. 2016, 95:1357-1366. 10.1016/j.ijrobp.2016.03.024

11. Barriger RB, Forquer JA, Brabham JG, et al.: A dose-volume analysis of radiation pneumonitis in non-small cell lung cancer patients treated with stereotactic body radiation therapy. Int J Radiat Oncol Biol Phys. 2012, 82:457-462. 10.1016/j.ijrobp.2010.08.056

12. Borst GR, Ishikawa M, Nijkamp J, et al.: Radiation pneumonitis after hypofractionated radiotherapy: evaluation of the LQ(L) model and different dose parameters. Int J Radiat Oncol Biol Phys. 2010, 77:15961603. 10.1016/j.ijrobp.2009.10.015

13. Inoue T, Oh RJ, Shiomi H, Masai N, Miura H: Stereotactic body radiotherapy for pulmonary metastases. Prognostic factors and adverse respiratory events. Strahlenther Onkol. 2013, 189:285-292. 10.1007/s00066012-0290-1

14. Ong CL, Palma D, Verbakel WF, Slotman BJ, Senan S: Treatment of large stage I-II lung tumors using stereotactic body radiotherapy (SBRT): planning considerations and early toxicity. Radiother Oncol. 2010, 97:431-436. 10.1016/j.radonc.2010.10.003

15. Bahig H, Filion E, Vu T, et al.: Severe radiation pneumonitis after lung stereotactic ablative radiation therapy in patients with interstitial lung disease. Pract Radiat Oncol. 2016, 6:367-374. 
10.1016/j.prro.2016.01.009

16. Matsuo Y, Shibuya K, Nakamura M, et al.: Dose--volume metrics associated with radiation pneumonitis after stereotactic body radiation therapy for lung cancer. Int J Radiat Oncol Biol Phys. 2012, 83:545-549.

10.1016/j.ijrobp.2012.01.018

17. Borst GR, Ishikawa M, Nijkamp J, et al.: Radiation pneumonitis in patients treated for malignant pulmonary lesions with hypofractionated radiation therapy. Radiother Oncol. 2009, 91:307-313.

10.1016/j.radonc.2009.02.003

18. Chang JY, Li QQ, Xu QY, et al.: Stereotactic ablative radiation therapy for centrally located early stage or isolated parenchymal recurrences of non-small cell lung cancer: how to fly in a "no fly zone". Int J Radiat Oncol Biol Phys. 2014, 88:1120-1128. 10.1016/j.ijrobp.2014.01.022

19. Marks LB, Bentzen SM, Deasy JO, et al.: Radiation dose-volume effects in the lung. Int J Radiat Oncol Biol Phys. 2010, 76:70-76. 10.1016/j.ijrobp.2009.06.091

20. Graham MV, Purdy JA, Emami B, Harms W, Bosch W, Lockett MA, Perez CA: Clinical dose-volume histogram analysis for pneumonitis after 3D treatment for non-small cell lung cancer (NSCLC). Int J Radiat Oncol Biol Phys. 1999, 45:323-329. 10.1016/\$0360-3016(99)00183-2

21. Okubo M, Itonaga T, Saito $\mathrm{T}$, et al.: Predicting risk factors for radiation pneumonitis after stereotactic body radiation therapy for primary or metastatic lung tumours. Br J Radiol. 2017, 90:20160508. $10.1259 /$ bjr.20160508

22. Yamashita H, Nakagawa K, Nakamura N, et al.: Exceptionally high incidence of symptomatic grade 2-5 radiation pneumonitis after stereotactic radiation therapy for lung tumors. Radiat Oncol. 2007, 2:21. 10.1186/1748-717X-2-21

23. Roberts SA, Hendry JH: The delay before onset of accelerated tumour cell repopulation during radiotherapy: a direct maximum-likelihood analysis of a collection of worldwide tumour-control data. Radiother Oncol. 1993, 29:69-74. 10.1016/0167-8140(93)90175-8

24. Fried DV, Das SK, Marks LB: Imaging radiation-induced normal tissue injury to quantify regional dose response. Semin Radiat Oncol. 2017, 27:325-331. 10.1016/..semradonc.2017.04.004

25. Selvaraj J, Lebesque J, Hope A, et al.: Modeling radiation pneumonitis of pulmonary stereotactic body radiotherapy: the impact of a local dose-effect relationship for lung perfusion loss. Radiother Oncol. 2019, 132:142-147. 10.1016/.radonc.2018.12.015

26. Benedict SH, Yenice KM, Followill D, et al.: Stereotactic body radiation therapy: the report of AAPM Task Group 101. Med Phys. 2010, 37:4078-4101. 10.1118/1.3438081

27. Wirsdorfer F, Jendrossek V: Modeling DNA damage-induced pneumopathy in mice: insight from danger signaling cascades. Radiat Oncol. 2017, 12:142. 10.1186/s13014-017-0865-1

28. Tsoutsou PG, Koukourakis MI: Radiation pneumonitis and fibrosis: mechanisms underlying its pathogenesis and implications for future research. Int J Radiat Oncol Biol Phys. 2006, 66:1281-1293. 10.1016/j.ijrobp.2006.08.058

29. Yamashita H, Kobayashi-Shibata S, Terahara A, et al.: Prescreening based on the presence of CT-scan abnormalities and biomarkers (KL-6 and SP-D) may reduce severe radiation pneumonitis after stereotactic radiotherapy. Radiat Oncol. 2010, 5:32. 10.1186/1748-717X-5-32

30. Chen H, Senan S, Nossent EJ, Boldt RG, Warner A, Palma DA, Louie AV: Treatment-related toxicity in patients with early-stage non-small cell lung cancer and coexisting interstitial lung disease: a systematic review. Int J Radiat Oncol Biol Phys. 2017, 98:622-631. 10.1016/j.ijrobp.2017.03.010 